\title{
Community-acquired Pediatric Urinary Tract Infections Caused by Morganella Morganii
}

\author{
[il Bahriye Atmış1, 囵 Soner Sertan Kara2, 回 Mehtap Hülya Aslan3 \\ ${ }^{1}$ Erzurum Regional Training and Research Hospital, Clinic of Pediatric Nephrology, Erzurum, Turkey \\ 2Erzurum Regional Training and Research Hospital, Clinic of Pediatric Infectious Disease, Erzurum, Turkey \\ ${ }^{3}$ Erzurum Regional Training and Research Hospital, Clinic of Microbiology, Erzurum, Turkey
}

\begin{abstract}
Aim: Escherichia coli is the most common microorganism recovered in urinary tract infections (UTI) in all age groups. Lately, different pathogens, such as Morganella morganii, are beginning to be isolated. The aim of this study was to investigate children with UTI caused by Morganella morganii.

Materials and Methods: Children with UTI caused by Morganella morganii in our pediatric outpatient clinic were retrospectively evaluated.

Results: The mean age of 11 children was $4.2 \pm 1.9$ years (minimum: 19 months, maximum: 7.5 years). Four (36.4\%) patients were female. The most frequent symptoms were irritability $(n=5,45.5 \%)$ and dysuria $(n=5,45.5 \%)$. Urinalysis was positive for leukocytes in $9(81.8 \%)$, hematuria in $5(45.5 \%)$, and nitrite in $6(54.5 \%)$ patients. None of the patients had electrolyte abnormalities or renal failure. Colony count was most prevalently 100,000 colony-forming unit $/ \mathrm{mL}(n=7,63.6 \%)$. The pathogen was most sensitive to imipenem/meropenem and piperacillintazobactam ( $n=11,100 \%$, for both). Two (18.1\%) patients were hospitalized. Empirical antibiotic treatments were switched to amikacin (15 mg/ $\mathrm{kg} /$ day) for outpatients and piperacillin-tazobactam (300 mg/kg/day) for hospitalized patients for 10 days. Repeat urine cultures on the third day of treatment were negative.

Conclusion: Morganella morganii, which is usually encountered as a kind of nosocomial or opportunistic infection, is presented as a cause of community-acquired UTI in this study. As in other infections, antibiotic susceptibility profiles are crucial in directing treatment .
\end{abstract}

Keywords: Children, community-acquired, morganella morganii, urinary tract infection

\section{Introduction}

Urinary tract infections (UTI) are among the most commonly observed infections in pediatric patients. As it may result in kidney damage and chronic renal failure, early recognition and treatment of a UTI are crucial. The prevalence of UTI was $7.0 \%$ among infants presenting with fever (1). The most commonly isolated microorganisms in all age groups are Gram-negative enteric bacteria such as Escherichia coli, Klebsiella, Proteus, Enterococcus or Enterobacter species (2).
Morganella morganii is a Gram-negative, facultative, anaerobic, non-lactose-fermenting, urease positive microorganism from the Enterobacteriaceae family which is found in the normal gastrointestinal flora. Like the other members of the Enterobacteriaceae family, M. morganii is naturally resistant to beta-lactam antibiotics (3). Although M. morganii is a common microorganism found in nature and in human habitats, it is rarely responsible for community-acquired infections. Instead, it often causes nosocomial infections such as postoperative infections, 
sepsis, soft tissue infections, meningitis, chorioamnionitis, endophthalmitis, arthritis or UTI (4-7). In a study conducted in Taiwan, out of 82,861 patients infected by Gram-negative microorganisms over a 6-year period, M. morganii was isolated from 1,219 (1.47\%) samples and ranked as the ninth most common nosocomial infection (8). In the recent years, there has been an increase in opportunistic infections caused by $M$. morganii, due to its enhanced virulence and antibiotic resistance (4). The aim of this study was to investigate pediatric patients with UTI caused by M. morganii, of which little is known regarding community-acquired infections.

\section{Materials and Methods}

Eleven pediatric patients (aged between 1 month and 18 years) who were diagnosed with UTI caused by Morganella morganii in the pediatric outpatient clinic of Erzurum Training and Research Hospital between January 15t, 2015, and August $1^{\text {st }}, 2017$, were retrospectively evaluated. Patient files were reviewed for patient age at time of diagnosis, presenting complaints, previous history of UTI, underlying diseases, laboratory and radiological results, and family history. The samples collected for urinalysis and culture were obtained using the appropriate technique according to patient age. In toilet-trained children, a mid-stream urine sample was obtained. In non-toilet-trained children, an adhesive urine collection bag was applied after disinfecting the skin in the genital area. If the urinalysis showed pyuria, urethral catheterization was performed to collect a urine sample for culture. All urine bag samples for urine culture were excluded. Children whose urinalyses were positive for leukocyte esterase and/or nitrite, bacteriuria, or pyuria were defined as UTI. Urine culture results of these patients were reviewed. A positive urine culture was defined as the growth of a single uropathogen microorganism of $>10^{5}$ colonyforming units $(\mathrm{CFU}) / \mathrm{mL}$ in a mid-stream urine sample or $>10^{4} \mathrm{CFU} / \mathrm{mL}$ in a urine sample obtained by catheterization (9). All patients were started on empiric treatment. Children who were treated as outpatients received oral cefixime $8 \mathrm{mg} / \mathrm{kg}$ once daily, whereas patients who were hospitalized were started on intravenous ceftriaxone 75 $\mathrm{mg} / \mathrm{kg} / \mathrm{day}$ in two divided doses. Then, the treatment was adjusted as necessary according to a determination of the sensitivity and resistance patterns of M. morganii. On the third day of antibiotic treatment, repeat urinalysis and urine culture samples were obtained from all patients to evaluate response to treatment. The duration of treatment was 10 days for all patients. All patients underwent renal and bladder ultrasonography (US). Dimercaptosuccinic acid (DMSA) scintigraphy was also performed at 4-6 months following the acute infection in patients with a history of recurrent UTI.

\section{Statistical Analysis}

Data was analyzed using the SPSS statistical software (SPSS for Windows, version 17.0; SPSS Inc., Chicago, IL, USA). The data were expressed as means and standard deviation (mean \pm standard deviation), number of patients ( $n$ ) and percentages (\%).

The study was approved by the Erzurum Regional Research and Training HospitalEthical committee with number 2018/03-17.

\section{Results}

During the study period, a total of 2,866 proven UTI were identified. M. morganii was isolated in urine cultures of 11 $(0.38 \%)$ patients. The mean age of the patients was $4.2 \pm 1.9$ years (minimum: 19 months, maximum: 7.5 years). Four patients (36.4\%) were female and 7 (63.6\%) were male. Two (18.1\%) patients had an underlying disease (asthma, operated congenital heart disease). The symptoms and laboratory findings of the patients are presented in Table I. Four patients (36.4\%) had a history of recurrent UTI. None of the patients had a family history of chronic kidney disease. Quantitative culture results showed 30,000 CFU/ $\mathrm{mL}$ in $3(27.3 \%)$ patients, $40,000 \mathrm{CFU} / \mathrm{mL}$ in $1(9.1 \%)$ patient, and $100,000 \mathrm{CFU} / \mathrm{mL}$ in 7 (63.6\%) patients. The pathogen was most sensitive to imipenem/meropenem ( $n=11,100 \%)$, piperacillin-tazobactam $(n=11,100 \%)$, and amikacin $(n=10$, 90.9\%); and most resistant to ampicillin and amoxicillinclavulanate (none of the strains were susceptible to these antibiotics). The properties of M. morganii strains isolated from the patients are presented in Table II.

None of the patients had visited another facility before

\begin{tabular}{|l|l|}
\hline \multicolumn{2}{|l|}{ Table I. Symptoms and laboratory findings of patients } \\
\hline \multicolumn{2}{|l|}{$\mathbf{n}(\%)$} \\
\hline Symptoms & $5(45.5)$ \\
\hline Irritability & $5(45.5)$ \\
Dysuria & $4(36.4)$ \\
Fever & $4(36.4)$ \\
Flank/abdominal pain & $4(36.4)$ \\
Nausea/vomiting & \\
\hline Laboratory findings & $9(81.8)$ \\
\hline Leukocyturia & $5(45.5)$ \\
Hematuria & $6(54.5)$ \\
Nitrite positivity & $6(54.5)$ \\
Leukocytosis & $2(18.2)$ \\
CRPa elevation & \\
\hline${ }^{a}$ CRP: C-reactive protein & \\
\hline
\end{tabular}


Table II. Properties of Morganella morganii strains isolated from patients

\begin{tabular}{|l|l|}
\hline Colony count (CFU/mL ${ }^{\mathbf{a}}$ ) & $\mathbf{n ~ ( \% )}$ \\
\hline 30,000 & $3(27.3)$ \\
40,000 & $1(9.1)$ \\
100,000 & $7(63.6)$ \\
\hline Antibiotic susceptibility rates & $\mathbf{n}(\%)$ \\
\hline $\begin{array}{l}\text { Imipenem/meropenem } \\
\text { Piperacillin-tazobactam }\end{array}$ & $11(100)$ \\
Amikacin & $11(100)$ \\
Trimethoprim/Sulfamethoxazole & $10(90.9)$ \\
Ceftriaxone & $8(72.7)$ \\
\hline aCFU/mL: number of colony forming units per milliliter, CFU: Colony-forming unit \\
\hline
\end{tabular}

presenting to our outpatient clinic. Two (18.1\%) patients were hospitalized due to decreased oral intake. The empirical treatment was modified according to sensitivity testing results. Those patients who were treated on outpatient follow-up ( $\mathrm{n}=9)$ were treated with intramuscular amikacin (15 $\mathrm{mg} / \mathrm{kg} /$ day), while hospitalized patients received intravenous piperacillin-tazobactam (300 mg/kg/day) for 10 days. Only a 19-month-old female patient (9\%) who was hospitalized had hydronephrosis and was diagnosed with unilateral hydronephrosis. DTPA (diethylenetriaminepentaacetic acid) scan of this patient revealed a partial ureteropelvic junction obstruction. No vesicoureteral reflux was observed in the voiding cystourethrogram of the patient. Four (36.4\%) of the patients underwent DMSA scintigraphy due to recurrent UTI and no abnormal findings were detected. Repeat urine cultures obtained on the third day were negative in all patients. During the ten-day follow-up, none of the patients developed any complications.

\section{Discussion}

In this study, M. morganii was investigated as a rare cause of UTI, which is a very common infection in the pediatric population. Since the 1930s when it was first described, M. morganii was thought to cause hospital-acquired infections (10). However, recent studies contradict this argument. It has also been reported to cause community-acquired infections such as UTIs, pyelonephritis, osteomyelitis, and peritonitis (11-13). Community-acquired UTIs caused by M. morganii have been reported as case reports. To our knowledge, this is the first study investigating communitybased UTIs caused by M. morganii in children.

There is a risk of developing urosepsis in the acute period, therefore pediatric UTIs are serious, especially in young children. It has been reported that in cases of bacteremia caused by $M$. morganii, the urinary tract was the most common source, followed by the hepatobiliary tract (4). The reported incidence of UTIs caused by M. morganii is between $1.6 \%$ and $37 \%$ (14). Contrary to the literature, M. morganii related UTIs were found less frequently than in other studies in our cohort. A history of hospital admission and surgical intervention have been described as risk factors for M. morganii bacteremia. However, infections other than UTI were reported to be independent risk factors for mortality (14). In previous studies, underlying chronic diseases have also been defined as risk factors for M. morganii bacteremia (15). In our study, none of the patients had any risk factors associated with hospital-acquired infections or had a clinical condition suggestive of bacteremia/sepsis. Therefore, blood cultures were not collected. In two patients who had chronic diseases, the UTI resolved without any complications after appropriate treatment. Given the fact that none of our patients had any risk factors, we believe that all our patients acquired the $M$. morganii infection from their natural environment and/or from their own gastrointestinal flora.

M. morganii is known to be intrinsically resistant to penicillins, first and second generation cephalosporins, macrolides, lincosamides, phosphomycin, fusidic acid and colistin. In our study, the pathogen was most susceptible to imipenem, meropenem, piperacillin-tazobactam and amikacin. These findings are in accordance with the study of Erlanger et al (14). However, Senel et al. (16), who described antibiotic resistance in pediatric UTIs, reported $100 \%$ susceptibility to imipenem but $13 \%$ resistance to piperacillin. The sensitivity rates of microorganisms to antibiotics may vary in time and with local epidemiological influencers. In our study, those who were treated as outpatients were given amikacin, whereas hospitalized patients received piperacillin-tazobactam, with good clinical response.

In our study, the mean age was $4.2 \pm 1.9$ years and $63.6 \%$ of the patients were male. According to the American Academy of Pediatrics (AAP), UTIs are 2-5 times more common in males younger than 3 months of age compared to females. In all age groups, symptomatic UTIs were 3-5 times more common in females than in males (17). In addition, UTIs are 5-20 times more common in uncircumcised males compared to circumcised males (18). In a study on 132 infants ( $<4$ months) with UTI, 68.9\% were male, whereas, in another study on 2,316 children with UTI, $50.9 \%$ were male $(19,20)$. The fact that the male to female ratio was greater than expected (with regard to age) in our study may stem from the fact that male patients in our study were not yet circumcised. In the region where the present study was conducted, most males are circumcised at a more advanced age. 
The most common symptoms of UTI among infants younger than 3 months of age are fever, vomiting, and irritability. In infants aged 3 months and older, fever is the most common symptom, followed by abdominal pain, flank pain, and vomiting. In older children, the most common symptoms are pollakiuria and dysuria, but enuresis, flank pain, abdominal pain, fever, nausea, strong smelling urine and cloudy urine may also be observed (21). In our study, the most common symptoms were irritability, dysuria, fever, flank/abdominal pain and nausea/vomiting, respectively.

In our study, $36.4 \%$ of patients had a history of UTI. However, no correlation was found between recurrent UTI and the growth of M. morganii in urine culture because none of the previous urine cultures of the patients were positive for M. morganii. The previous UTI episodes of the patients had taken place from between 2 months to 1 year previously. None of the patients had required hospitalization during their previous UTI.

None of the patients included in our study had a family history of chronic renal disease. The sensitivity of leukocyte esterase in the detection of UTI is between $83-94 \%$, whereas the specificity of nitrite testing is $90-100 \%$. A positive result for leukocyte esterase or nitrite combined with a positive urine microscopy result increases the sensitivity to $99.8 \%$ (22). The urinalysis findings of our patients were, in decreasing order, leukocyturia, hematuria and nitrite positivity.

Leukocytosis and elevated CRP levels, which indicate acute inflammation and infection, were observed in 54.5\% and $18.2 \%$ of patients, respectively. In a study on children with UTI, $11.9 \%$ of patients had normal leukocyte counts and CRP levels (23). In our study, $45.5 \%$ of patients had normal CRP and leukocyte levels. This is presumably due to the fact that none of the patients included in our study presented with pyelonephritis.

Aside from urosepsis, UTI may lead to numerous other complications. If not treated timely and appropriately, UTI may cause severe dehydration and hypoperfusion in the short term and result in acute kidney injury (24). Additionally, in the long term, the renal scarring that can develop may result in hypertension and chronic kidney disease (24). In our study, the BUN, creatinine and electrolyte levels at the time of admission and during follow-up were within normal limits. As a result, none of the patients suffered from any complications.

When evaluating patients with UTIs, radiological imaging modalities are used to detect underlying urologic anomalies and to confirm acute pyelonephritis. In the NICE (National
Institute for Health and Care Excellence) guidelines, urinary system US is recommended for infants with UTI who are $<6$ months of age. On the other hand, the AAP guideline recommends US for all children with febrile UTI, regardless of age $(17,22)$. In a series of 309 children under 2 years of age, $88 \%$ of the patients had normal urinary system US results (25). Similarly, $91 \%$ of the children in our study had normal urinary US results.

\section{Study Limitatitons}

Our study has some limitations which must be mentioned. The limitations of this study include the small sample size and only having data from a single center.

\section{Conclusion}

An important point to notice is that even though $M$. morganii is part of the normal gastrointestinal flora and mostly causes nosocomial and opportunistic infections in patients with predisposing factors, it was isolated as a cause of community-acquired UTI in this study. The patient's clinical condition and the antibiotic susceptibility profile are important in the planning of treatment.

\section{Ethics}

Ethics Committee Approval: The study was approved by the Erzurum Regional Research and Training Hospital Ethical committee with number 2018/03-17.

Informed Consent: Retrospective study.

Peer-review: Externally peer-reviewed.

\section{Authorship Contributions}

Concept: B.A., S.S.K., M.H.A., Design: B.A., S.S.K., M.H.A., Data Collection or Processing: B.A., S.S.K., Analysis or Interpretation: B.A., S.S.K., M.H.A., Literature Search: B.A., S.S.K., M.H.A., Writing: B.A., S.S.K., M.H.A.

Conflict of Interest: The authors have no conflicts of interest relevant to this article to disclose.

Financial Disclosure: The authors have no financial relationships relevant to this article to disclose.

\section{References}

1. Shaikh N, Morone NE, Bost JE, Farrell MH. Prevalence of urinary tract infection in childhood: a meta-analysis. Pediatr Infect Dis ) 2008;27:302-8.

2. Gür D, Kanra G, Ceyhan M, Seçmeer G, Kanra B, Kaymakoğlu I. Epidemiology and antibiotic resistance of gram-negative urinary pathogens in pediatric patients. Turkish / Pediatrics 1999;41:37-42. 
3. O'Hara CM, Brenner FW, Miller JM. Classification, identification, and clinical significance of Proteus, Providencia, and Morganella. Clin Microbiol Rev 2000;13:534-46.

4. Liu H, Zhu J, Hu Q, Rao X. Morganella morganii, a non-negligent opportunistic pathogen. Int / Infect Dis 2016;50:10-7.

5. Johnson JR, Feingold M. Case of chorioamnionitis in an immunocompetent woman caused by Morganella morganii. J Matern Fetal Med 1998;7:13-4.

6. Tsanaktsidis G, Agarwal SA, Maloof AJ, Chandra ), Mitchell P. Postoperative Morganella morganii endophthalmitis associated with subclinical urinary tract infection. / Cataract Refract Surg 2003;29:1011-3.

7. Cetin M, Ocak S, Kuvandik G, Aslan B, Temiz M, Aslan A. Morganella morganii-associated arthritis in a diabetic patient. Adv Ther 2008;25:240-4.

8. Chen YT, Peng HL, Shia WC, et al. Whole-genome sequencing and identification of Morganella morganii KT pathogenicityrelated genes. BMC Genomics 2012;13Suppl 7:S4.

9. Hodson EM, Craig IC. Urinary tract infections in children. In: Avner ED, Harmon WE, Niaudet P, Yoshikawa N, Emma F, Goldstein SL (eds). Pediatric Nephrology. Seventh Edition. Berlin, Springer-Verlag, 2016:1695-714.

10. Lin TY, Chan MC, Yang YS, et al. Clinical manifestations and prognostic factors of Morganella morganii bacteremia. Eur J Clin Microbiol Infect Dis 2015;34:231-6.

11. Nasri YM, Denden RI, Guo Q, Mastouri M, Aouni M, Wang M. Type II and type IV topoisomerase mutations in clinical isolates of Morganella morganii harbouring the qnrD gene. Ann Clin Microbiol Antimicrob 2014;13:34.

12. Koyuncu S, Ozan F. Morganella morganii osteomyelitis complicated by secondary septic knee arthritis: a case report. Acta Orthop Traumatol Turc 2012;46:464-7.

13. Atalay H, Güney I, Solak Y, Almaz E. First case of CAPDrelated peritonitis caused by Morganella morganii. Perit Dial Int 2010;30:119-21.

14. Erlanger D, Assous MV, Wiener-Well Y, Yinnon AM, Ben-Chetrit E. Clinical manifestations, risk factors and prognosis of patients with Morganella morganii sepsis. I Microbiol Immunol Infect 2019;52:443-8.
15. Falagas ME, Kavvadia PK, Mantadakis E, et al. Morganella morganii infections in a general tertiary hospital. Infection 2006;34:315-21.

16. Senel S, Karacan C, Erkek N, Gol N. A single-center experience of antimicrobial resistance patterns in pediatric urinary tract infection. Med Princ Pract 2010;19:359-63.

17. Roberts KB. Urinary Tract Infection: Clinical Practice Guideline for the Diagnosis and Management of the Initial UTI in Febrile Infants and Children 2 to 24 Months. American Academy of Pediatrics. Committee on quality improvement and management subcommittee on urinary tract infection and steering. Pediatrics 2011;595-610.

18. Goldberg B, Jantausch B. Urinary Tract Infection. In: Kher KK, Schnaper HW, Greenbaum LA (eds). Clinical Pediatric Nephrology Third Edition. Baco Raton, CRC press, 2017:967-91.

19. $\mathrm{Wu} \mathrm{JH}$, Chiou $Y \mathrm{H}$, Chang JT, Wang HP, Chen YY, Hsieh KS. Urinary tract infection in infants: a single-center clinical analysis in southern Taiwan. Pediatr Neonatol 2012;53:283-8.

20. Wang J, He L, Sha J, et al. Etiology and antimicrobial resistance patterns in pediatrics with urinary tract infections. Pediatr Int 2018;60:418-22.

21. Downs SM. Technical Report: urinary tract infections in febrile infats and young children. Pediatrics 1999;103:1-60.

22. National Institute for Health and Clinical Excellence. NICE clinical guideline 54. Urinary tract infection in children: Diagnosis, treatment and long-term management.2007; pp.136.

23. Tamgumus S, Geoghan J, Coghlan D, Nadeem M. Urinary Tract Infection in Childhood and Inflammatory Markers. Ir Med I 2016;109:442.

24. Practice parameter: the diagnosis, treatment, and evaluation of the initial urinary tract infection in febrile infants and young children. American Academy of Pediatrics. Committee on Quality Improvement. Subcommittee on Urinary Tract Infection Pediatrics. 1999;103(4 Pt 1):843-52.

25. Hoberman A, Charron M, Hickey RW, Baskin M, Kearney DH, Wald ER. Imaging studies after a first febrile urinary tract infection in young children. N Engl I Med 2003;348:195-202. 